\title{
TÉCNICA DE MODELAGEM NUMÉRICA PELO MÉTODO DOS ELEMENTOS FINITOS PARA ESTUDO DA RESPOSTA ACÚSTICA VEICULAR
}

\author{
Tiago Simão Ferreira ${ }^{1}$ \\ Pedro Américo Magalhães Junior ${ }^{2}$ \\ Claysson Bruno Santos Vimieiro ${ }^{3}$ \\ Frederico Luiz de Carvalho Moura ${ }^{4}$ \\ Marco Antônio Mendonça Vecci ${ }^{5}$ \\ Gabriel Oliveira Ribeiro ${ }^{6}$
}

\begin{abstract}
RESUMO
Este estudo aborda a técnica de modelagem da resposta acústica de um veículo automotor. Esta pesquisa discorre sobre a construção de modelos numéricos em elementos finitos para investigar o efeito da utilização de componentes móveis (capô, portas laterais e tampa do porta malas) na resposta acústica de um veículo. Para tanto, foram construídos dois modelos: um que abrange a utilização de todos os componentes móveis, e outro, que substitui cada componente móvel por elementos unidimensionais (1D), contendo apenas a massa e a inércia que representa cada um destes componentes. A resposta obtida em cada uma das etapas da simulação numérica é comparada com os resultados experimentais, a fim de validar o procedimento e compreender a significância da utilização de um modelo numérico simplificado e assim, validar um modelo simplificado capaz de reproduzir computacionalmente e de forma satisfatória a resposta acústica de um veículo. A utilização do referido modelo simplificado possibilita a indústria automotiva realizar estudos sobre a performance acústica de um veículo de forma antecipada, uma vez que não necessita esperar o desenvolvimento destes componentes. Como investigado nesta pesquisa, a influência de componentes móveis sobre a resposta acústica do modelo numérico completo é pequena quando comparado à resposta obtida pelo modelo simplificado. Por meio de uma segunda
\end{abstract}

\footnotetext{
${ }^{1}$ Mestrado em Engenharia Mecânica pela Pontifícia Universidade Católica de Minas Gerais (PUC-Minas) e docente do Instituto Federal de Minas Gerais - Campus Congonhas. E-mail: tiago.simao@ifmg.edu.br.

${ }^{2}$ Doutorado em Engenharia Mecânica e mestrado em Engenharia Elétrica pela Pontifícia Universidade Católica de Minas Gerais (PUC-Minas). E-mail: paamj@oi.com.br.

3 Pós-doutorado na área de Propriedade Intelectual, doutorado e mestrado em Engenharia Mecânica pela Universidade Federal de Minas Gerais (UFMG). Docente do Programa de Pós-graduação em Engenharia Mecânica da Pontifícia Universidade Católica de Minas Gerais (PUC-Minas). Coordenador do Núcleo de Sistemas Mecânicos do Departamento de Engenharia Mecânica e Mecatrônica da Pontifícia Universidade Católica de Minas Gerais (PUC-Minas). E-mail: claysson@gmail.com.

${ }^{4}$ Mestrando em Engenharia de Estruturas e graduação em Engenharia Mecânica pela Universidade Federal de Minas Gerais (2005). E-mail: frediluiz@yahoo.com.br.

${ }^{5}$ Mestrado em Engenharia Aeronáutica e Mecânica pelo Instituto Tecnológico de Aeronáutica (ITA) e docente adjunto do Departamento de Engenharia de Estruturas da Escola de Engenharia da Universidade Federal de Minas Gerais (UFMG). E-mail: vecci@dees.ufmg.br.

${ }^{6}$ Graduado em Engenharia Civil pela Universidade Federal de Minas Gerais (UFMG), especialização e mestrado em Engenharia de Estruturas, doutorado em Engenharia Civil Estruturas pela Universidade de São Paulo (USP São Carlos). Pós-doutorado na Vanderbilt University em Nashville, Estados Unidos (USA). Professor titular do Departamento de Engenharia de Estruturas da Universidade Federal de Minas Gerais (UFMG). E-mail: gabriel@dees.ufmg.br.
} 
análise, "Participação de Painéis", é visto que a contribuição na resposta acústica final dos componentes móveis somados é inferior a $3 \mathrm{~dB}$.

Palavras-chave: Método dos Elementos Finitos. Análise Acústica. Participação de Painéis. Acústica Veicular.

\title{
NUMERICAL MODELING TECHNIQUE USING THE FINITE ELEMENT METHOD IN VEHICLE ACOUSTIC RESPONSE STUDY
}

\begin{abstract}
This study examines a modeling technique of the acoustic response in automotive vehicle. This research focuses on the development of numerical models in finite elements that investigate the effect of mobile components (hood, side doors and trunk lid) in the vehicle acoustic response. For this, two finite element models were constructed: one that encompasses the use of all the mobile components, and other that replace each component (hood side doors and trunk lid) by one-dimensional (1D) elements, containing only the mass and inertia that represent each component. The response obtained in each step of the numerical simulation is compared to the experimental results in order to validate the procedure and understand the significance of using a simplified numerical model. It is expected, at the end of the research that the validation of a simplified model is able to reproduce, computationally and satisfactorily the acoustic response of a vehicle. This simplified model enables the automotive industry to conduct studies on the acoustic performance of a vehicle in advance, once, it does not require waiting to develop these components. Thus, as investigated in this research, the influence of mobile components on the acoustic response of the complete numerical model is small when compared to the response obtained by the simplified model. By a second analysis, "Panels Participation" it was observed that the contribution at the final acoustic response of mobile components summed is less than $3 \mathrm{~dB}$.
\end{abstract}

Keywords: Finite Element Method. Acoustic Analysis. Panel Participation Technique. Vehicular Acoustics.

\section{INTRODUÇÃO}

Nos dias atuais, ruído, vibração e aspereza (NVH) desempenham um papel importante para a definição dos requisitos funcionais de um veículo moderno e, diante de um novo cenário de uma exigência cada vez maior por parte dos clientes, um dos desafios do mercado de automóveis é fornecer um veículo eficiente e com alta qualidade percebida. Hoje em dia, um dos critérios mais importantes para a avaliação da qualidade de um veículo é baseado em níveis de emissão acústica, vinculado ao nível de ruído transmitido de fora para dentro do veículo ou pelo ruído produzido pelo próprio veículo. Consequentemente, existe uma atenção 
geral para conceber os critérios que visam melhorar o comportamento acústica-estrutural, de modo a suportar os padrões de exigência dos clientes cada vez mais restritivos, (BRAESS, 2005; FERREIRA; MOURA; MAGALHÃES, 2013).

Diante do exposto, esta pesquisa busca validar uma ferramenta numérica capaz de satisfazer as necessidades da indústria automotiva, no sentido de elaborar estudos na área de acústica veicular e estabelecer uma metodologia de simulação. Com o objetivo de estudar o comportamento do modelo numérico de elementos finitos, este trabalho centra-se na aplicação e validação de métodos computacionais que possam prever o comportamento acústico e estrutural do veículo completo. Os testes desta pesquisa foram focados em veículos compactos (veículos tais como fabricados no Brasil).

Para este estudo foram construídos dois modelos de elementos finitos: um que abrange a utilização de todos os componentes móveis e outro que substitui cada componente (capô, portas laterais e tampa da mala) por elementos unidimensionais (1D), contendo apenas a massa e a inércia que os representa. Feito isto, os resultados numéricos foram comparados com os resultados testados em laboratório. Em geral, este tipo de estudo é feito até uma frequência próxima de $300 \mathrm{~Hz}$, devido a limitações de processamento (MOURA et al., 2012; PRIEBSCH et al., 2001). Entretanto, este estudo tem como objetivo determinar, por meio da verificação do grau de correlação, o processamento dos modelos até a frequência de $500 \mathrm{~Hz}$.

Finalmente, buscando validar um modelo numérico simplificado capaz de reproduzir computacionalmente e de forma satisfatória a resposta acústica de um veículo, este estudo está dividido em duas partes, a saber: A primeira seria os testes físicos de veículos reais e simulações numéricas de modelos computacionais, em seguida, a partir da apresentação dos resultados gerados, realizar o estudo de correlação entre os resultados obtidos.

\section{METODOLOGIA}

\subsection{Procedimentos Experimentais}

A primeira etapa deste trabalho foi a seleção de um projeto (veículo) para a sequência dos estudos da pesquisa. Nesse caso, foi utilizado um veículo sedan compacto, que foi escolhido por representar uma gama de veículos compactos fabricados no Brasil. Após a escolha do veículo, alguns estudos foram realizados em laboratório, utilizando câmara acusticamente isolada para medições experimentais de pressão sonora geradas por excitações 
estruturais. A fase experimental foi desenvolvida tanto para fornecer um conjunto de medidas de referência para as atividades numéricas, quanto para avaliar os níveis de pressão sonora gerados dos vários pontos de excitações estruturais. Nesta fase foram escolhidos, como pontos de excitações estruturais, os pontos de fixação do motor à carroceira. Estes foram escolhidos porque são os pontos de maior entrada de energia à carroceira na condição de utilização usual do veículo em condição de tráfego urbano.

Para a primeira parte, o veículo sedan compacto selecionado foi analisado em câmara acusticamente isolada (ambiente acusticamente controlado) e, com um martelo de impacto, os pontos de fixação do motor foram excitados. Para cada "martelada" proferida sobre estes pontos, foram feitas medições com o uso de um microfone colocado à altura da orelha direita do motorista, e assim, recolhidos os dados fornecidos de pressão sonora.

A figura 1 esclarece os pontos de excitações estruturais usados pela equipe de testes experimentais. Nesta figura temos o ponto de excitação um (1), sendo o ponto de fixação do motor à carroceria; o ponto de excitação dois (2), sendo o ponto de fixação do cambio à carroceria e o ponto de fixação (3) sendo o ponto de fixação da bieleta do motor à carroceria. O impacto gerado por um martelo foi realizado no ponto imediatamente adjacente ao posicionamento do acelerómetro apresentado na figura 1 (lado direito).
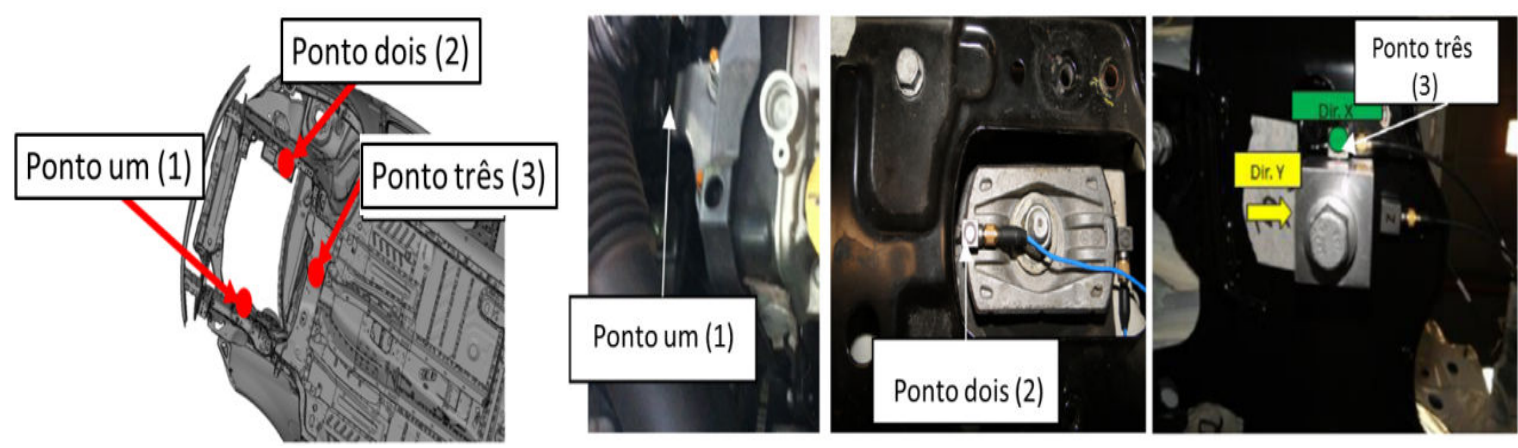

Figura 1 - Configuração do modelo numérico (lado esquerdo) e experimental (lado direito) - vista inferior do veículo (pontos 1, 2 e 3)

Fonte: Autor.

Após a definição dos pontos de excitação, o ponto de medição acústica descrito acima é apresentado na figura 2, (FIAT AUTO, 2004). Nesta figura, é apresentado o ponto de fixação do microfone na altura da orelha direita do condutor (o lado esquerdo da figura) e detalhamento do posicionamento do microfone (lado direito da figura). 

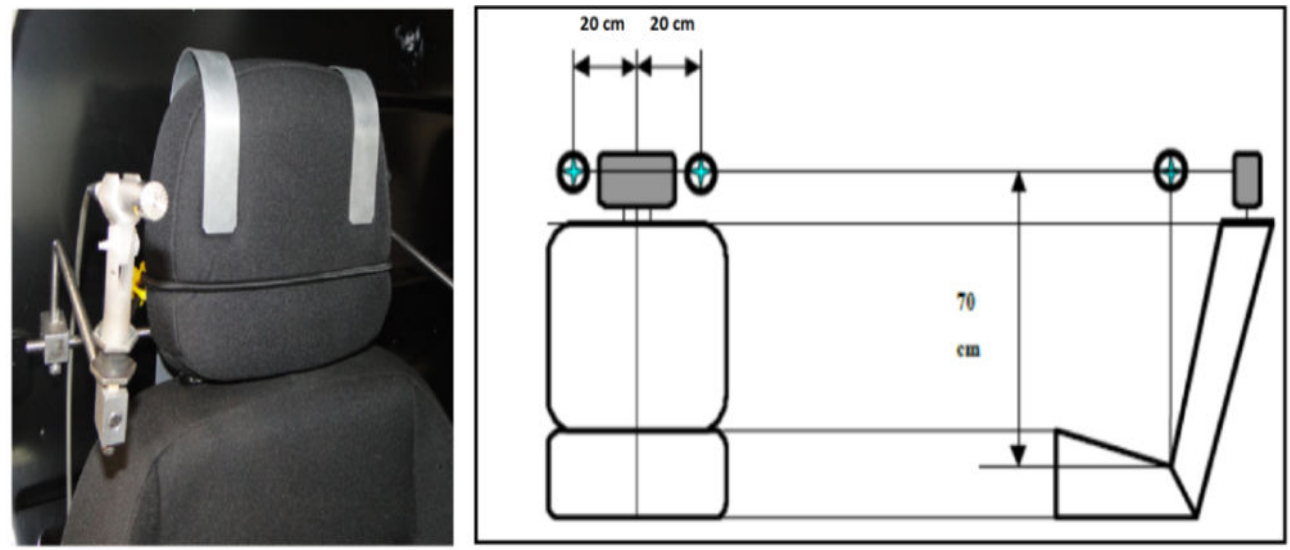

Figura 2 - Configuração experimental do ponto de montagem do microfone (ponto de coleta de dados) Fonte: Autor.

Em relação à coleta de dados experimentais, o ensaio do veículo foi realizado numa câmara acusticamente isolada, usando o equipamento LMS Test Lab 11B (PEETERS et al., 2001). A tabela 1 mostra a cadeia de equipamento usado para as medições experimentais.

Tabela 1 - Cadeia de equipamentos

\begin{tabular}{cc}
\hline Equipamentos & Sensibilidade / Detalhes \\
\hline Martelo de impacto & $2 \mathrm{mV} / \mathrm{N}$ \\
Microfone & $50 \mathrm{mV} / \mathrm{Pa}$ \\
LMS Test Lab & Scadas Mobile - Modulo Impact Testing \\
\hline
\end{tabular}

Fonte: Autor.

\subsection{Procedimentos numéricos}

Na sequência, dois modelos de veículos virtuais foram criados em elementos finitos. A diferença entre os modelos está na forma como os componentes móveis (capô, portas laterais e tampa da mala) foram modelados.

Assim, para criar os modelos de elementos finitos da carroceria, um pacote comercial foi adotado (software Altair HyperMesh 11.0). A tabela 2 descreve as características que compõem cada modelo virtual (completo e simplificado). 
Tabela 2 - Características dos modelos numéricos

\section{Características dos modelos numéricos}

\begin{tabular}{ll}
\hline Modelo 1 - Veículo completo & Modelo com todos os componentes. \\
Modelo 2 - Elementos modelos com & Janelas, radiador, para-choques \\
massa e inércia concertados (Versão & dianteiro e traseiro, Portas, Porta \\
simplificada) & malas, capô, tanque de combustível e \\
& faróis dianteiros e traseiros. \\
Todos os modelos - Componentes não & Suspensão dianteira e traseira, sistema \\
modelados. & de descarga, motor. \\
\hline
\end{tabular}

Fonte: Autor.

O veículo, agora modelado numericamente, é avaliado medindo a sua resposta no ponto indicado na figura 2, com base nas excitações estruturais feitas nos pontos indicados pela figura 1. Abaixo, temos a configuração para o layout do veículo "carroceria completa" usada para os estudos de sensibilidade acústica. Na figura 3 é possível ver a versão dos dois modelos numéricos, uma vez que a parte (a) de tal figura apresenta o modelo numérico completo que utiliza todos os componentes móveis (componentes com coloração mais escura) devidamente modelos e a parte (b), uma versão simplificada, onde cada item descrito como “componentes móveis” são representados por sua massa e inércia.

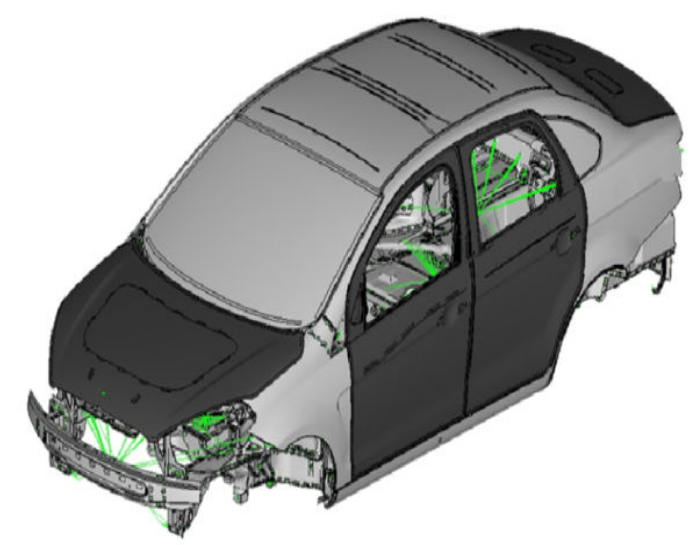

(a) Modelo1: com partes móveis

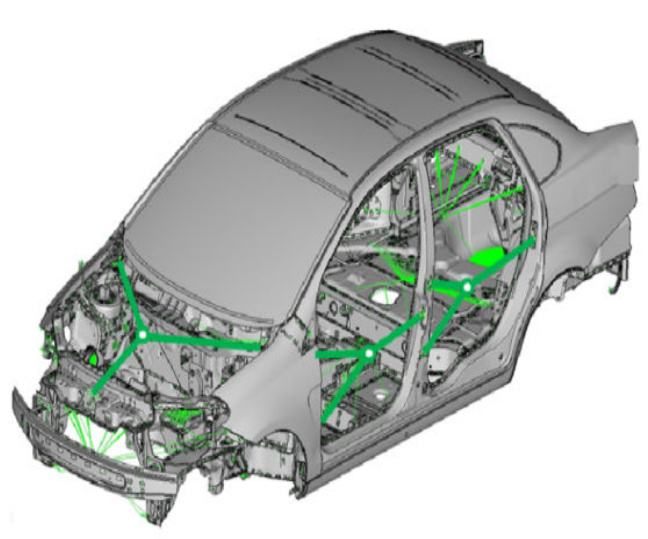

(b) Modelo 2: Versão simplificada, usando dados de inércia e massa.

Figura 3 - Configuração do modelo completo, modelos em elementos finitos (MEF) Fonte: Autor.

Observe que a figura 3 apresenta a montagem de componentes móveis em diferentes configurações. 
A construção dos dois modelos numéricos foi feita utilizando o pacote comerical da Altair, o HyperMesh 11.0, um software específico de pré-processamento. Ambos os modelos têm sua construção composta por elementos do tipo casca (elementos planos com três ou quatros nós) e por elementos sólidos (Elementos tri-dimensionais com cinco, seis ou oito nós). Para a solução modal estrutural, foi utilizado o algoritmo LANCZOS (DAVIDSSON; SANDBERG, 2006) para os cálculos de autovalores e autovetores.

Após a construção dos modelos numéricos estruturais dos veículos, foi construido o modelo interno da cavidade. Para a construção desta cavidade foram usados a representação dos painéis de instrumentos e o volume dos bancos (isto considerando a sua densidade). A figura 4 apresenta os detalhes da construção em elementos finitos da cavidade interna do modelo do veículo. Esta cavidade, acoplada aos dois modelos apresentados na figura 3, foi usada para calcular o nível de pressão sonora (SPL).

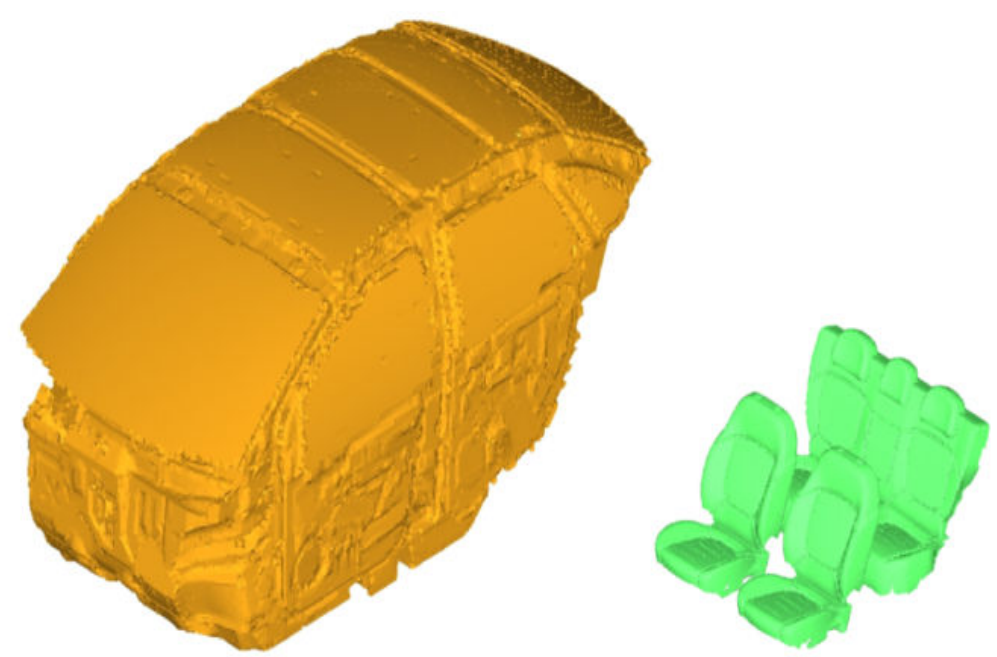

Figura 4 - Representação do modelo de cavidade interno do veículo e o detalhamento dos bancos Fonte: Autor.

O detalhamento da construção dos modelos estuturais e de cavidade estão apresentados na tabela 3 abaixo. 
Tabela 3 - Composição MEF do modelo Estrutural e de Cavidade

\begin{tabular}{lll}
\hline Detalhes da malha MEF & & \\
\hline Modelo MEF (1) & Modelo MEF (2) & Fluído MEF \\
\hline Massa: 21 & Massa: 27 & Elementos 5 nós: 264724 \\
Solda:6259 & Solda:6016 & Elementos 6 nós: 185788 \\
Rigidos:8706 & Rigidos:540 & Elementos 6 nós: 224815 \\
RBE3:26 & RBE3:26 & \\
Molas: 1 & Molas:1 & \\
Elementos 3 nós: 27961 & Elementos 3 nós: 19849 & \\
Elementos 4 nós: 1004699 & Elementos 4 nós: 712229 & \\
Elementos 6 nós: 5117 & Elementos 6 nós: 4277 & \\
Elementos 8 nós: 110536 & Elementos 8 nós: 94012 & \\
& & \\
\hline Total elementos: 1163326 & Total elementos: 836977 & Total elementos: 675327 \\
Total nós: 1146344 & Total nós: 877224 & Total nós: 453703 \\
\hline Fonte: Autor. & &
\end{tabular}

No modelo de elementos finitos que representa a cavidade, um nó específico foi selecionado com interesse especial. É sobre este nó que são obtidos as respostas de pressão sonora (SPL). Este nó é escolhido de acordo com seu posicionamento espacial e em conformidade com a posição do microfone usada para os testes exeperimentais e detalhado na Figura 2.

Após ter sido realizado a "solução 103" no processamento do modelo de cavidade, por meio do Nastran, fez-se o acoplamento (Estrutura - Fluido) através da solução 111 do Nastran (KOMZSIK, 2001). Então, usando VEIPROD 5.0.2 ${ }^{\circledR}$ (software para avaliação da FRF - análise de SPL) para o processamento destes dados, a resposta acústica interna do veículo é gerada. De posse dos resultados acústicos preliminares e da demarcação das áreas dos modelos numéricos que compõem os painéis de interesses para esta pesquisa, o software VEIPROD 5.0.2 ${ }^{\circledR}$ gera os resultados da análise de "Participação de painéis". O resultado mostra, em termos percentuais, a quantidade que cada um dos painéis contribui para a resposta acústica numa dada frequência.

Para este estudo é importante construir o modelo numérico do veículo como um conjunto de painéis para saber, numericamente, a importância ou a influência que cada painel exerce na resposta acústica final (aqui podemos dizer que cada componente móvel se comporta como um painel). A matriz que gera, pelo software VEIPROD, a solução numérica para a resposta acústica é: 


$$
\left[\begin{array}{cc}
-M \omega^{2}+K & -A^{T} \\
-\omega^{2} A & -M_{f} \omega^{2}+K_{f}
\end{array}\right]\left\{\begin{array}{l}
x \\
p
\end{array}\right\}=\left\{\begin{array}{l}
F \\
0
\end{array}\right\}
$$

onde cada termo da matriz é representada da seguinte forma:

$[\mathrm{M}]=$ matriz de massa estrutural; $[\mathrm{K}]$ = matriz de rigidez estrutural; $[\mathrm{A}]=$ Matriz de superfície de contato; $[\mathrm{Mf}]=$ matriz "em massa" do fluido ; $[\mathrm{Kf}]=$ Matriz de "rigidez" do fluido $; x\}=$ Vetor de deslocamentos estrutural; $\{\mathrm{P}\}=$ Vetor de pressões sonora; $\{\mathrm{F}\}=$ vetor de forças aplicadas.

No entanto, a resposta final acústica (SPL) para este estudo é normalizada pela força usada no ponto de excitação, para que possamos comparar os resultados numéricos e experimentais sem a influência dessa variável. Em referência aos autores Citarella, Frederico e Cicatiello (2007), utiliza-se o termo "Sensibilidade Acústica de Carroceria" (SAC) para se referir a esta função de transferência de vibro-acústica.

O SAC é definido como a relação entre o nível de pressão sonora $\mathrm{P}(\omega)$ no interior do corpo da carroceria (num ponto específico i) e o nível de excitação do f estrutural ( $\omega$ ) gerado (aplicado no ponto $\mathrm{j}$ ), expressa $\mathrm{em}[\mathrm{dB} / \mathrm{N}]$ :

$$
S A C(\omega)_{i, j}=20 \log _{10}\left(\frac{p(\omega)_{i}}{f(\omega)_{j}}\right)
$$

Para a análise acústica, as propriedades do fluido que representa o modelo de cavidade são as seguintes, tabela 4:

Tabela 4 - Propriedades do fluido (MEF)

\begin{tabular}{cc}
\hline \multicolumn{2}{c}{ Propriedades do fluido } \\
\hline Densidade: & $1.22 \mathrm{~kg} / \mathrm{m}^{3} ;$ \\
Velocidade do som: & $344 \mathrm{~m} / \mathrm{s} ;$ \\
Pressão de referência: & $2 \mathrm{E}-0.5 \mathrm{~N} / \mathrm{m}^{2}$. \\
\hline
\end{tabular}

Fonte: Autor.

Assim, até aqui, foram apresentados os procedimentos de medição experimental usados como referências de correlação com os resultados numéricos, e também, os detalhes de construção dos modelos numéricos usados para caracterizar um veículo sedan. Tais detalhes foram utilizados tanto para apresentar um modelo de veículo "completo" com todas as partes móveis (figura 3(a)), como também apresentar um modelo simplificado, onde as partes móveis foram substituídas por elementos unidimensionais (figura 3(b)). 
$\mathrm{Na}$ sequencia, serão apresentados os resultados individuais e suas correlações com os resultados experimentais.

\section{RESULTADOS}

Com base nas configurações mostradas anteriormente, tanto para os modelos de simulação (MEF) como para a configuração experimental, obtivemos os resultados de SAC, mostrados na figura 5 .

$\mathrm{Na}$ figura 5, a curva preta representa a resposta acústica do veículo. Segundo dados experimentais, a linha vermelha representa o resultado do modelo de elementos finitos da versão simplificada, em outras palavras, utilizando apenas os dados de inércia e de massa. $\mathrm{O}$ resultado apresentado pela linha azul representa a curva de resposta acústica do modelo completo em relação à utilização de componentes móveis.
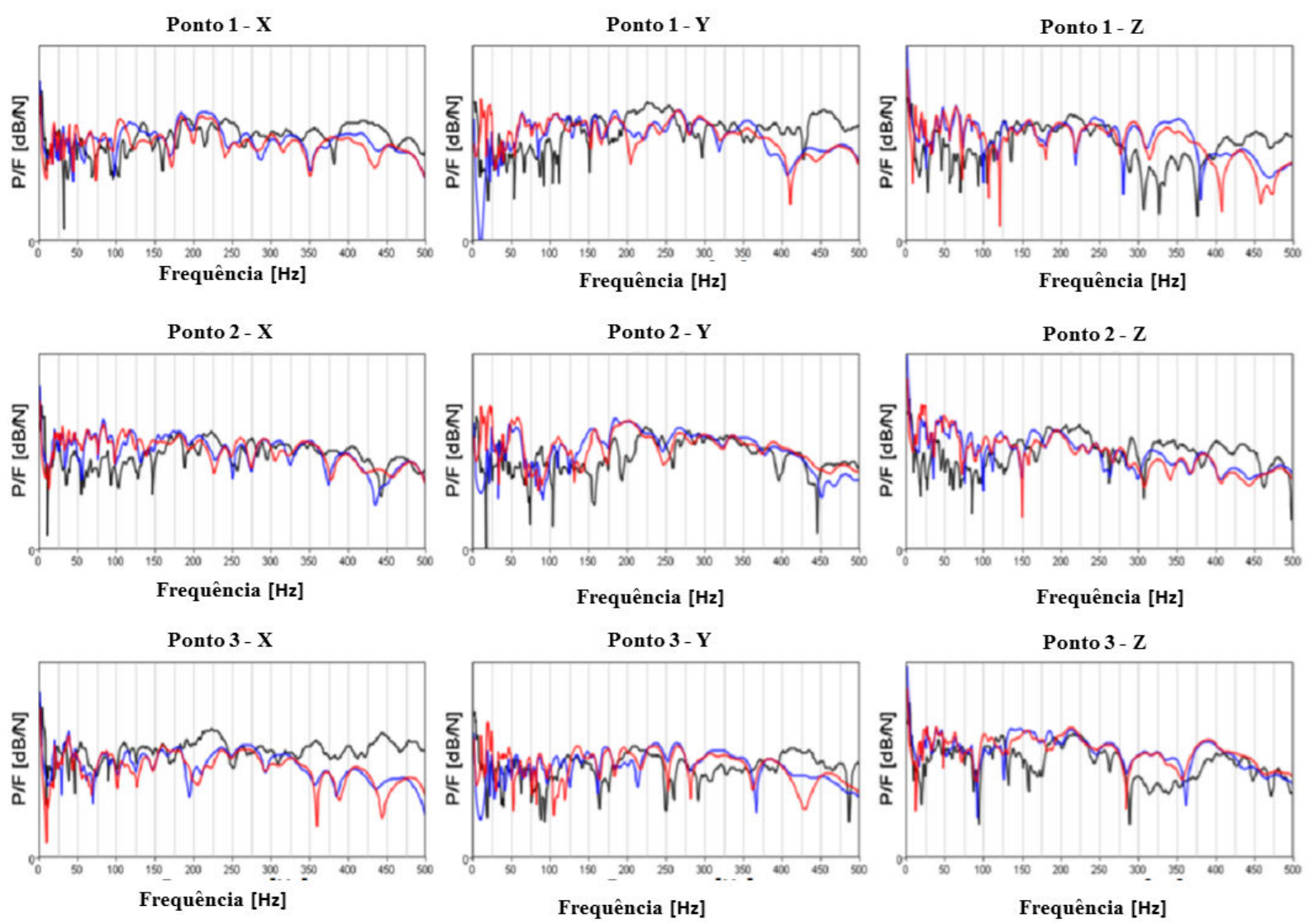

Figura 5 - Resultado de sensibilidade acústica da carroceria (Curva azul: Modelo completo; Curva vermelha: Modelo simplificado; Curva preta: resultado experimental)

Fonte: Autor. 
Cumpre informar que, os resultados apresentados na figura 5 foram obtidos com base nas excitações feitas nos pontos de 1, 2, 3 e nas direções cartesianas X, Y, Z. Estes pontos e direções estão ilustrados na figura 1 e suas respectivas respostas medidas no ponto que representa a posição espacial do ouvido direito de um condutor, conforme mostrado na figura 2.

Como podemos ver, os resultados numéricos e experimentais mostram uma boa correlação entre as faixas de frequência analisadas. Por meio desta análise, pode-se ver, dentro da faixa de frequência que representa o estudo sobre ruído estrutural, como os resultados gerados pelos dois modelos são semelhantes, tendo uma maior variação apresentada entre 450 e $500 \mathrm{~Hz}$.

$\mathrm{E}$, dos resultados apresentados na figura 5 é possível observar que estes apresentam boa correlação quando comparado com os resultados experimentais (linha preta). Este ótimo desempenho se destaca principalmente no ponto 2 . O ponto 3 apresenta uma boa correlação nas direções $\mathrm{Y}$ e $\mathrm{Z}$, porém com um resultado não tão satisfatório para a direção $\mathrm{X}$, principalmente acima de $300 \mathrm{~Hz}$. O desvio nos resultados pode ser explicado por se tratar de um ponto mais flexível e, portanto, mais complexo de ser representado numericamente.

Além dos resultados apresentados na figura 5 e na busca de uma melhor compreensão do efeito da contribuição das partes móveis, foi feito, por meio da análise de "Participação de Painéis", o estudo que seria um impacto sobre os resultados finais de acústica (SAC) quando se utiliza a representação em elementos finitos das partes móveis. Assim, conforme apresentado na figura 6, podemos ver a influência dos componentes móveis usando esta técnica sobre os resultados de acústica gerados do ponto 1 na direção $Z$.

A curva verde apresenta os resultados gerados pelo modelo completo (figura 3(a)) A curva vermelha utiliza este mesmo modelo. Porém, por meio de recurso computacional, foi retirada a influência das partes móveis (destacado em cinza na figura 3(a)). O termo "recurso computacional" refere-se à remoção da influência da matriz de massa $[\mathrm{M}]$ e rigidez [k] fornecida no pós-processamento do modelo completo da figura 3(a). 


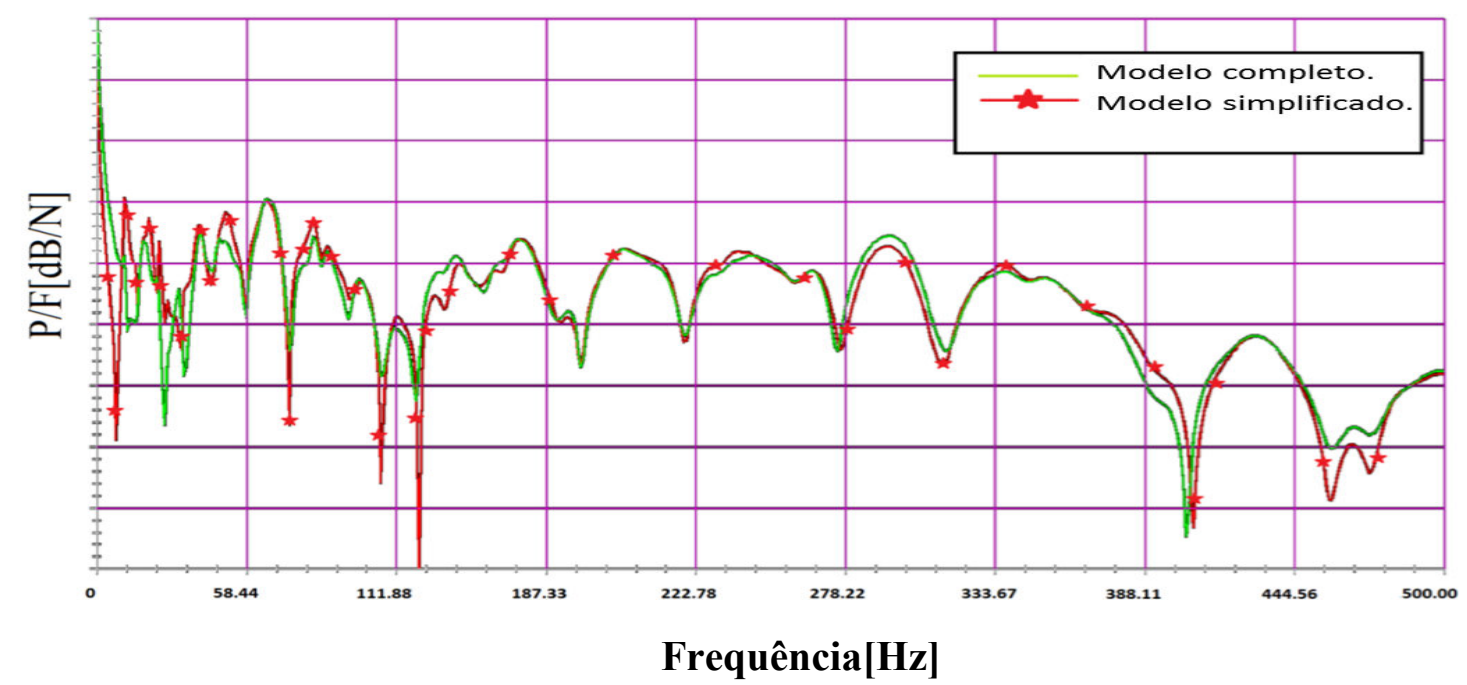

Figura 6 - Sensibilidade acústica da carroceria (O espaçamento no eixo ordinário é de $10 \mathrm{~dB}$ ) Fonte: Autor.

Por meio de análise da figura 6 , podemos ver que a resposta gerada sem a participação da matriz de massa $[\mathrm{M}]$ e rigidez $[\mathrm{K}]$ dos componentes móveis (curva vermelha) difere do resultado original (curva verde), principalmente na faixa de frequências entre $450 \mathrm{~Hz}$ e $500 \mathrm{~Hz}$. Assim, foi realizado um estudo sobre este veículo para a compreensão plena da influência dos componentes móveis (capô, portas laterais e tampa do porta malas) pela "Participação de Painéis" no qual a resposta total acústica do veículo é analisada, especificamente, na frequência de $475 \mathrm{~Hz}$ (frequência pontual onde se observou a maior diferença entre os resultados).

Na figura 7 podemos ver a distribuição, medida em escala logarítmica, gerada do ponto 1, na direção "Z", da participação de cada painel do veículo (teto, assoalho, parede corta fogo etc.) nos resultados acústicos finais, avaliados na frequência de $475 \mathrm{~Hz}$. 


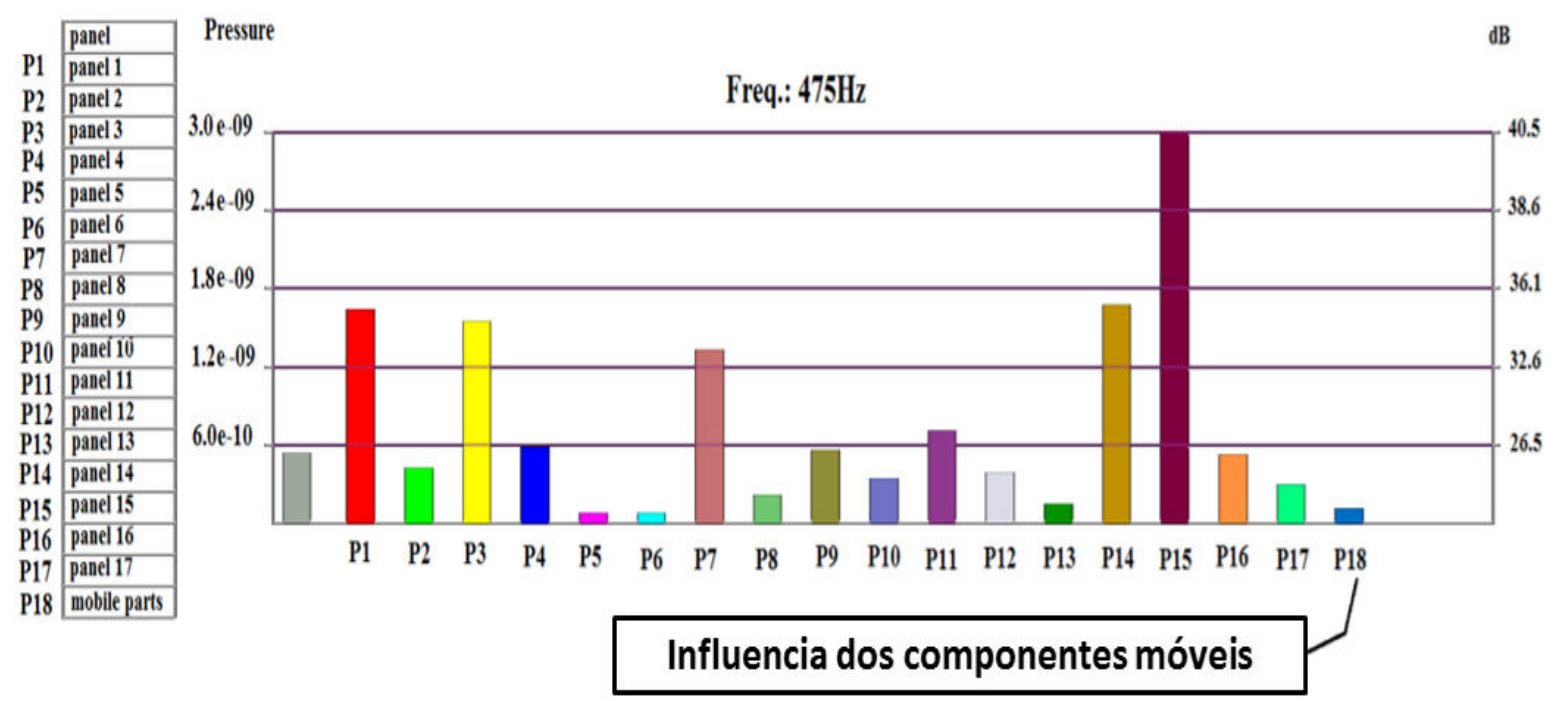

Figura 7 - Resultados de SAC da análise de participação de painéis Fonte: Autor.

Pela análise do gráfico de "Participação de Painéis" da figura 7, pode-se ver a influência dos componentes móveis em modelos veiculares sobre a resposta acústica, avaliadas na frequência de maior discrepância dos resultados $(475 \mathrm{~Hz})$ apresentado no estudo da figura 6.

Assim, pode-se analisar que o impacto da utilização de "componentes móveis", apresentados na figura 3(a), na construção de modelos completos é pequeno, quando comparado com outros painéis. Os componentes móveis, que somados estão representados pelo ID "P18" na figura 7, apresentam uma influência menor do que 5\% na resposta total do sistema acústico (SAC).

\section{CONCLUSÃO}

Nesta experiência observou-se que o método dos elementos finitos pode apresentar uma boa correlação no uso da avaliação do desempenho acústico-estrutural numa faixa de frequência de 0 à $500 \mathrm{~Hz}$, uma vez que o modelo é representativo quando comparado com os dados experimentais.

O destaque deste trabalho é a análise de que a influência de componentes móveis é inferior a $5 \%$, portanto exercem uma pequena influência na resposta acústica do modelo numérico. Também, por meio de uma segunda análise, "Participação de Painéis", vimos que a contribuição na resposta acústica final dos componentes móveis, representados pelo ID "P18" na figura 7 é muito pequena quando comparado a outros painéis. 
No entanto, este estudo é centrado na utilização de veículos compactos, na qual os componentes móveis não permitem a existência de painéis de grandes dimensões ou flexíveis, como no caso de veículos comerciais. Neste caso, sugere-se um estudo específico.

Assim, é possível concluir que é possível obter bons resultados acústicos em uma fase preliminar de projetos automotivos, trabalhando no desenvolvimento de carros compactos, como o usado no presente artigo, onde ainda nem todos os componentes móveis foram desenvolvidos. Isso permitirá os analistas agirem com antecedência nos estudos de problemas acústicos.

Finalmente, o estudo considera válido, como uma boa alternativa, a abordagem baseada no uso de versões de modelos simplificados, a exemplo a figura 3(b), como um procedimento de simulação acústica veicular pelo método dos elementos finitos.

\section{AGRADECIMENTOS}

O autor agradece, gentilmente, pelo suporte fornecido: Pontifícia Universidade Católica de Minas Gerais - PUCMINAS, a Coordenação de Aperfeiçoamento de Pessoal de Nível Superior - CAPES, a Fundação de Amparo a Pesquisa de Minas Gerais - FAPEMIG, ao Conselho Nacional de Desenvolvimento Cientifico e Tecnológico - CNPq, ao Instituto Federal de Minas Gerais - IFMG e, também, a FIAT Automóveis do Brasil e Altair do Brasil.

\section{REFERÊNCIAS}

BISTAFA, S. R. Acústica aplicada ao controle do ruído. 2. ed. São Paulo: Edgard Blucher, 2012.

BRAESS, H. H; SEIFFERT, U. Handbook of automotive engineering. New York: SAE International, 2005.

CITARELLA, R.; FREDERICO, L.; CICATIELLO, A. Modal acoustic transfer vector approach in a FEM-BEM vibro-acoustic analysis. Engineering Analysis with Boundary Elements, [S.1], v. 31, n. 3, p. 248-258, 2007. DOI: 10.1016/j.enganabound.2006.09.004.

DAVIDSSON, P.; SANDBERG, G. A reduction method for structure-acoustic and poroelastic-acoustic problems using interface-dependent lanczos vectors. Computer Methods in Applied Mechanics and Engineering, [S.1], v. 195, n. 17-18, p. 1933-1945, 2006. DOI:10.1016/j.cma.2005.02.024. 
FERREIRA, T. S.; MOURA, F.; MAGALHÃES, P. Sensitivity analysis of numerical and experimental comparison by nvh finite element simulation in "trimmed body" to different excitation points of a vehicle in the frequency range until 500hz. In: INTERNATIONAL CONGRESS OF MECHANICAL ENGINEERING (COBEM), 22., 2013, Ribeirão Preto. Anais... Rio de Janeiro: ABCM, 2013. ISSN 2176-5480.

FIAT AUTO. Performance Standard 7- R0151: vehicle and shell: acoustic/vibration transfer functions analysis. [S.1]: Satiz-Normazione, 2004.

KOMZSIK, L. MSC/NASTRAN numerical methods: user's guide, version 68. Los Angeles: Macneal-Schwendler Corp., 2001.

MOURA, F. et al. Numerical and experimental comparison by NVH Finite Element Simulation in "Body in White" of a vehicle in the frequency range until $800 \mathrm{~Hz}$. In: SAE BRASIL INTERNATIONAL NOISE AND VIBRATION COLLOQUIUM, 2012, Florianópolis. Proceedings... Warrendale, US: SAE International, 2012. SAE Technical Paper 2012-36-0629. DOI:10.4271/2012-36-0629.

PEETERS, B. et al. A new procedure for modal parameter estimation. Sound And Vibration, Leuven, Belgium, p. 24-28, jan. 2004. Disponível em:

$<$ http://www.sandv.com/downloads/0401 peet.pdf>. Acesso em: 07 jul. 2014.

PRIEBSCH, H. H. et al. Numerical Simulation of Vehicle Interior Noise up to $250 \mathrm{~Hz}$. In: STYRIAN NOISE, VIBRATION \& HARSHNESS CONGRESS, 2001, Graz, Austria. [Proceedings...]. Graz: [s.n.], 2001, p. 149-161. Disponível em: $<$ https://online.tugraz.at/tug_online/voe_main2.getVollText?pDocumentNr=42424\&pCurrPk $=23358>$. Acesso em: 07 jul. 2014 .

Recebido em: 22/ 09/2014

Aprovado em: 16/12/2014

Publicado em: 29/06/2015 